\title{
Geleitwort zu „Rehabilitationsstandards für die Anschlussheilbehandlung und allgemeine Rehabilitation für Patienten mit einem Herzunterstützungssystem (VAD Ventricular Assist Device)“
}

\author{
Hermann Reichenspurner ${ }^{1}$
}

Online publiziert: 16. Februar 2016

(C) Springer-Verlag Berlin Heidelberg 2016

Die Therapie mit mechanischen Kreislaufunterstützungssystemen hat in den letzten Jahren einen deutlichen Zuwachs erlebt. Dies hat verschiedene Ursachen.

Die Goldstandard-Therapie der terminalen Herzinsuffizienz ist nach wie vor die Herztransplantation. Die Überlebensraten sind exzellent, mit 1-Jahresdaten von über $90 \%$ und 5-Jahresdaten von $75 \%$. Auch die Lebensqualität der transplantierten Patienten ist als sehr gut einzustufen. In Deutschland allerdings ist die Zahl der Herztransplantationen in den letzten Jahren deutlich rückläufig und letztes Jahr wurden weniger als 300 Herztransplantationen vorgenommen. Die Hauptursache liegt im akuten Spendermangel, insbesondere im europäischen Vergleich (BRD: 10 Organspender/Mio Einwohner, Österreich: 24 Organspender/Mio Einwohner, Spanien: 32 Organspender/Mio Einwohner!). Aufgrund der speziellen Gesetzeslage in Deutschland und der negativen Presse, die in den letzten Jahren veröffentlicht wurde, wird sich dies in absehbarer Zeit auch nicht verbessern. Somit steigt die Anzahl der Patienten auf der Warteliste stetig, wir benötigen in Deutschland dringend alternative therapeutische Verfahren.

Die Therapie mit mechanischen Kreislaufunterstützungssystemen hingegen hat in den letzten Jahren deutlich zugenommen und so wurden 2014 über 900 Herzunterstützungssysteme implantiert. Hier hat es einen enormen technischen Fortschritt gegeben und die neuesten implantierbaren Systeme der dritten Generation sind inzwischen voll implantierbar und müssen nur noch über ein dünnes Kabel nach außen mit einer Energiequelle verbunden werden. Auch die häufigsten Komplikationen nach VAD Implantation, wie z. B. Schlaganfall oder Infektionen des Systems sind zwar nach wie vor vorhanden, aber in den letzten Jahren deutlich rückläufig gewesen. Die Überlebensraten sind in den ersten beiden Jahren denen nach Herztransplantation durchaus vergleichbar.

In den nächsten Jahren wird sich das Indikationsspektrum zur Transplantation gegenüber den Herzunterstützungssystemen verändern, mehr Patienten werden einer primären VAD-Implantation zugeführt werden. Entscheidend hier ist eine gute Aufklärung der Patienten und eine sorgfältige Nachbetreuung insbesondere unmittelbar nach dem Krankenhausaufenthalt im Rahmen einer Rehabilitation. Aus diesem Grunde ist es besonders wichtig, dass vom Arbeitskreis VAD unter Federführung der SchüchtermannKlink hier ein Positionspapier zu den Rehabilitationsstandards nach VAD Implantation erarbeitet wurde und ich beglückwünsche die Autoren $\mathrm{zu}$ diesem hervorragenden Manuskript.

Hermann Reichenspurner

Hamburg, den 21.12.2015

Prof. Dr. med. Hermann Reichenspurner Ph.D

reichenspurner@uke.de

Universitäres Herzzentrum Hamburg, Hamburg, Deutschland 